\title{
A measure of confidence for Phase-Based Motion Estimator applied to 2D US-TO images
}

\author{
$\underline{\text { Emilia Badescu }^{1}}$, Sébastien Salles $^{2}$, Denis Friboulet ${ }^{1}$, Hervé Liebgott $^{1}$ \\ ${ }^{1}$ Univ Lyon, INSA-Lyon, Université Claude Bernard Lyon 1, UJM-Saint Etienne, CNRS, Inserm, CREATIS UMR 5220, U1206, \\ F-69100, LYON, France \\ ${ }^{2}$ Department of Circulation and Medical Imaging, Norwegian University of Science and Technology, Norway
}

Email: emilia.badescu@creatis.insa-lyon.fr

\begin{abstract}
Recent studies showed the potential of the Phase Based Motion Estimator when applied to ultrasound images containing transverse oscillations. Even though the emergence of ultrafast or high frame rates imaging overcame some of the prior limitations of the algorithm regarding its capability to detect large displacement values, the method still presents an important lack of robustness. In this paper, we propose an improvement of the Phase Based Motion Estimator, by introducing a measure of confidence.

The robustness of the method is indeed highly influenced by the orientations of the patterns present in radio frequency images containing transverse oscillations. The current phase based method relies on a specific pattern, containing pixels oriented in the two perpendicular directions of the oscillations. Any deviation from the assumed image model results in a underperforming estimate. The detection of the orthogonal patterns, which are in concordance with the theoretical image model, could guarantee the favorable outcome of the algorithm. A promising tool for identifying the desired patterns is given by Gabor filters oriented in 0 and 90 degrees direction. The magnitude response of the filter to the image orientations can be then grouped in two classes by applying the k-Means method. The class described by high intensity values is expected to guarantee the accuracy of the motion estimator. By keeping the regions corresponding with the first class and discarding the ones identified as belonging to the second class, we eliminate the underperforming estimates and increase the robustness of the method. The validation on simulated ultrafast images improved estimates with error reduced by $73 \%$ in the axial direction and $53 \%$ in the lateral direction, compared to the case when no measure of confidence was used.
\end{abstract}

Keywords- Ultrasound, Transverse oscillations, Motion estimation, Plane waves, Gabor filters.

\section{INTRODUCTION}

Many studies focused on motion estimation applied to Ultrasound (US) images due to its potential and wide range of applications in medical diagnosis. Most of the methods presented in literature can be classified as being a part of two main groups: amplitude based and phase based methods. Speckle tracking is representative for the first category and it involves finding the best possible match between two consecutive frames. The disadvantage of the amplitude based methods, consisting in the instability to the contrast and intensity variations that characterize the US images can be overcome by the phase based methods. As showed in [1], the accuracy is indeed significantly improved when phase based methods such as multivector and monogenic algorithms are used.

The limitation of most of the motion estimation algorithms applied to conventional US images is linked to the poor accuracy of the estimator in the direction perpendicular to the beam axis. The feature determining the good performance in the axial direction is the presence of the oscillations in the direction of the US beam. This yields to the idea of introducing the same type of oscillations in the direction perpendicular to the beam axis (the transverse direction), in order to obtain high accuracy along both axes. This approach was firstly exploited in blood flow imaging by Jensen and Munk [2] and by Anderson [3]. Transverse oscillations (TO) were then also introduced in elastography [4],[5] and echocardiography [6].

As shown in the comparison presented by Alessandrini et. al. [7], different phase based methods combined with TO outperform the block matching method applied to images without TO in terms of accuracy of the estimator. The potential of another phase based motion estimator (PBME) applied to US-TO images has also been revealed in previous studies from our team [7]. However, the method still presents some limitations. One of them corresponds to the fact that estimating a displacement greater than half of the wavelength is, by design, not possible. As shown by Salles et al. [8], the problem can be overcome by using ultrafast imaging.

The second limitation corresponds to the lack of robustness. The conventional PBME relies on the so-obtained bidirectional patterns in the image and yields an estimated displacement in a given region of interest (ROI) by averaging the individual displacements estimated for each pixel of that region. This may lead to an unreliable result when the patterns in the ROI deviate from the assumed model, increasing the standard deviation of the estimated displacements. Several studies addressed this problem by adapting RF images formation in order to improve the quality of the desired bidirectional pattern in each ROI [9]. 
In this paper, we propose a different approach based on adapting the motion estimator by discarding pixels that do not present the desired pattern. Gabor filters represent a promising tool for such analysis, which motivates its utilization as a measure of confidence.

The principles of TO, PBME and our measure of confidence based on Gabor filters are provided in Section II. In Section III, the results associated with those methods are exposed and discussed. Finally, the conclusion is presented in Section IV.

\section{METHODS}

\section{A. TOs by filtering}

The oscillations that are naturally present in the direction of the beam favor the accuracy of motion estimation in the axial direction. The lack of oscillations in the lateral direction impedes from achieving the same performance for the transversal displacement estimation. A natural approach for solving this problem would be to introduce transverse oscillations for RF ultrasound images. One possibility, as proposed by Salles et. al. [8], would be to generate TO by filtering the beamformed RF image. This approach is exploited in this study by using a spatial filter, expressed as a multiplication between a Gaussian and a sinusoid function [8]:

$$
\omega(x)=G(x) \cdot \cos \left(2 \pi f_{x_{0}} x\right)
$$

where $x$ represents the lateral position, $\omega(x)$ represents the spatial lateral filter and $\mathrm{G}(x)$ is the Gaussian function.

This filtering is performed in the Fourier domain by multiplying each row belonging to the 2D Fourier Transform of the RF image, with the Fourier Transform of $\omega(x)$. The resulting $2 \mathrm{D}$ spectrum contains four components, which are symmetrical with respect to the two spatial frequencies directions (axial and lateral, respectively).

\section{B. Phase based motion Estimator}

The four single quadrant spectra allow obtaining both the lateral and axial displacement components. Due to the symmetry in the Fourier domain, the phase of two of the four analytical signals calculated for each quadrant spectrum is sufficient for estimating the displacement values.

The displacement estimation is performed by computing two phase differences (each difference being related to the phases corresponding to each single quadrant spectrum) between two consecutive images, as described by Basarab et al. [10] and expressed hereunder:

$$
d_{z}=\frac{\Phi_{\text {diff }}+\Phi_{\text {diff }}}{2 \pi f_{z_{0}}}
$$

$$
d_{x}=\frac{\Phi_{\text {diff }}-\Phi_{\text {diff }}}{2 \pi f_{x_{0}}}
$$

Where $d_{z}, d_{x}$ correspond to the axial and lateral displacements, $\Phi_{\text {diff }_{1}}, \Phi_{\text {diff }_{2}}$ represent the phase differences of the first and second analytical signals computed for the initial and the displaced image and $f_{z_{0}}, f_{x_{0}}$ are the axial and the lateral spatial frequencies $\left(m^{-1}\right)$.

\section{The measure of confidence}

The PBME algorithm yields an estimated displacement in a given region of interest (ROI) by averaging the individual displacements estimated for each pixel of that region. The accuracy of the method relies on the underlying structure of the RF-TO images, that contain oscillations oriented in 0 and 90 directions. Each deviation from this pattern yields an erroneous estimate for each pixel, which affects the overall average value.

Detecting the regions oriented in the directions of the oscillations corresponds to the detection of the areas where the algorithm proves the highest accuracy. A suitable tool to detect patterns having a specific orientation is given by the Gabor filter having the following expression:

$g(x, y ; \lambda, \theta, \sigma, \gamma)=\exp \left(-\frac{x^{\prime 2}+\gamma^{2} y^{\prime 2}}{2 \sigma^{2}}\right) \cos \left(\frac{2 \pi x^{\prime}}{\lambda}\right)$

where $x^{\prime}=x \cos \theta+y \sin \theta$ and $y^{\prime}=-x \sin \theta+y \cos \theta, \quad \lambda$ represents the wavelength of the sinusoidal term, $\theta$ allows setting the desired orientation to be detected, $\sigma$ is the standard deviation of the Gaussian and $\gamma$ is the spatial aspect ratio.

The patterns oriented in the desired directions respond with high intensity values to the filter and the patterns having different orientations provide lower intensity responses. Thus, the response to the Gabor filter can be interpreted as a two class intensity map. These two classes can be determined by using k-Means classification method. The algorithm partitions the pixels into $\mathrm{k}=2$ classes, by minimizing the sum of squared differences between each pixel intensity value and the mean intensity value corresponding to cluster $\mathrm{k}$. At the end, the class containing low intensity values can be then discarded in order to improve the robustness of the algorithm.

A step by step illustration of the algorithm can be observed in Fig. 1. Note that the region of interest is quantized before using the Gabor filters, in order to minimize the impact of the intensity variation within a ROI on the Gabor magnitude. From each margin of the image, a number of pixels equivalent to half of the wavelength is replaced by 0 , in order to minimize the border effect. 


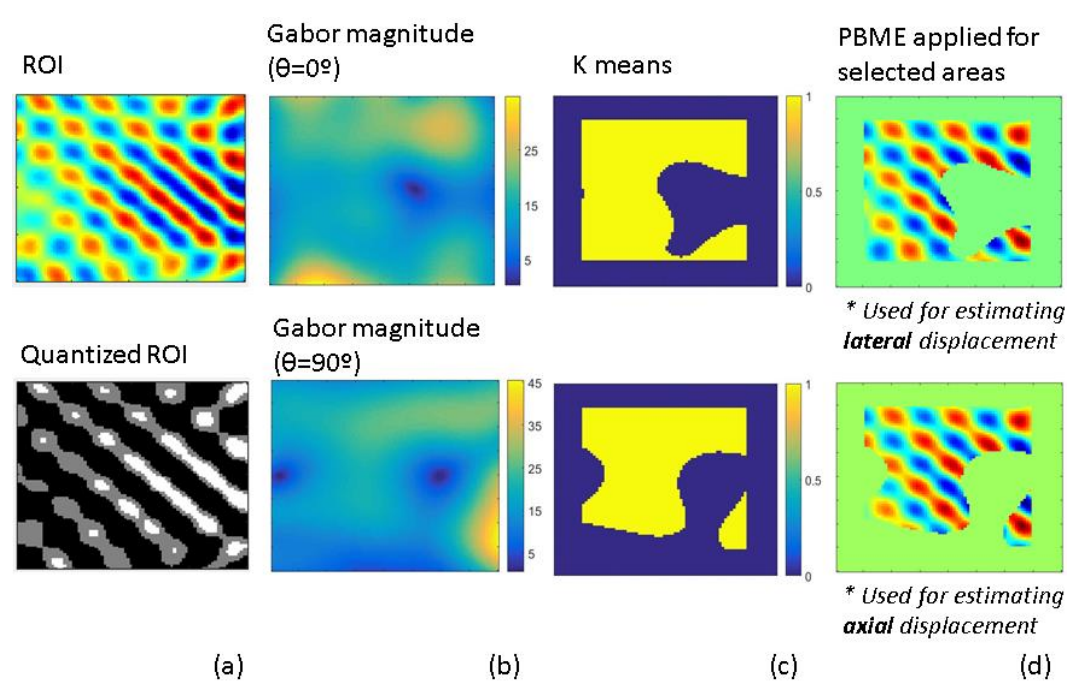

Fig. 1. Step by step Method. (a) Initial and quantized ROI. (b) The responses to 0 degrees and 90 degrees oriented Gabor filters (c) The two classes determined by using the k means algorithm. (d) Selected region to apply PBME

\section{RESULTS}

The algorithm was tested on images simulated using Field II [11], [12]. A 50x50 $\mathrm{mm}^{2}$ phantom composed of 8000 scatterers was imaged by using a 64 elements linear transducer. The central frequency of the transducer was set to $5 \mathrm{MHz}$ and a sampling frequency of $100 \mathrm{MHz}$ was used. The width of each element was set to $215 \mu \mathrm{m}$ and the kerf to 30 $\mu \mathrm{m}$, giving a pitch of $245 \mu \mathrm{m}$. The phantom was displaced using the realistic trajectory given in Fig. 2. The mean interframe displacements associated to these trajectories and their ranges are presented in Table 1.

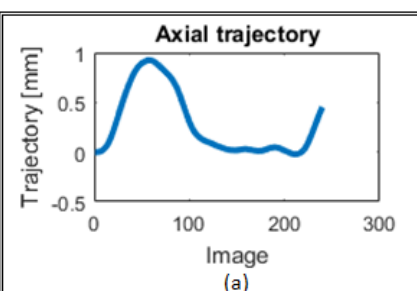

(a)

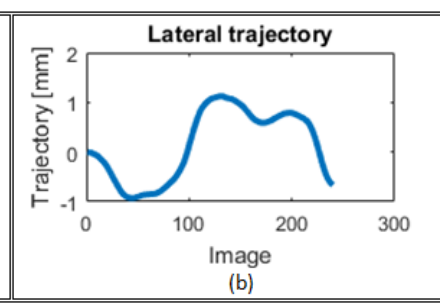

Fig. 2. (a) Axial and (b) Lateral reference trajectories
TABLE I. REAL VALUES USED FOR INTER-FRAME DISPLACEMENT: MEAN AND RANGE FOR A SEQUENCE OF 240 IMAGES

\begin{tabular}{|c|c|c|}
\hline $\begin{array}{l}\text { Displacement } \\
\text { values }\end{array}$ & Axial & Lateral \\
\hline Mean & $1.324 \mathrm{pxl}(10.2 \mu \mathrm{m})$ & $0.088 \mathrm{pxl}(21.6 \mu \mathrm{m})$ \\
\hline Range & $\begin{array}{c}0.0045 \mathrm{pxl}-4.0260 \mathrm{pxl} \\
(35 \mathrm{pm}-31 \mu \mathrm{m})\end{array}$ & $\begin{array}{c}2.0408 \cdot 10^{-7} \mathrm{pxl}-0.3094 \mathrm{pxl} \\
(0.05 \mathrm{pm}-75.8 \mu \mathrm{m})\end{array}$ \\
\hline
\end{tabular}

As stated in [13], PBME is unable to follow a motion higher than half of the wavelength, which motivated our choice to use a trajectory that presents small displacement values. However, the estimation of displacements lower than half of a wavelength may not be sufficient to detect a motion corresponding to the realistic values describing the motion in the human tissue. In order to apply the algorithm capabilities to realistic displacement values, one possibility would be to acquire data at high frame rate. In this study, we used the approach for plane wave compounding proposed by Montaldo et al. [14]. A number of 16 angles were used to generate the images.

In order to show the robustness of the method, the algorithm was validated on 20 sequences, each of them containing 240 images following the trajectory illustrated in Fig. 2. The histograms of axial and lateral errors are shown in Fig. 3 and 4 respectively. It can be observed that the standard deviation in the axial and in the lateral direction was lower when the measure of confidence had been used prior to PBME, compared to the conventional case.

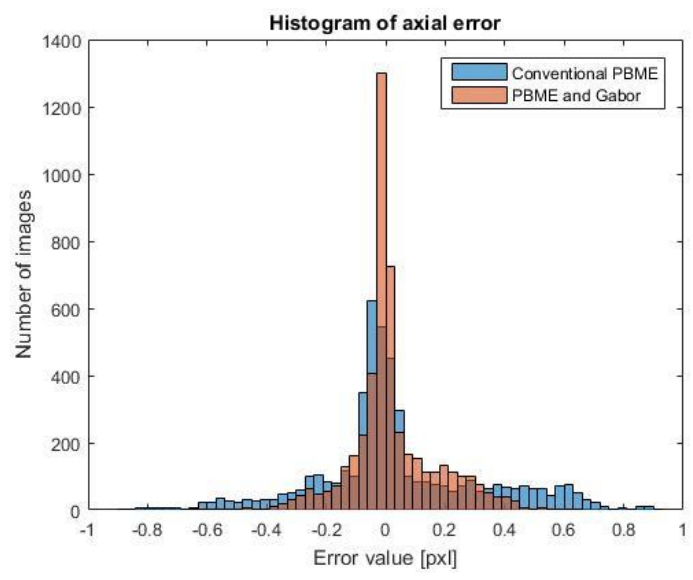

Fig. 3. Histogram of error [Axial]. The resulting histogram of error by using PBME and Gabor filters (orange) shows a lower standard deviation compared to the histogram of error obtained by using the conventional PBME method (blue). 


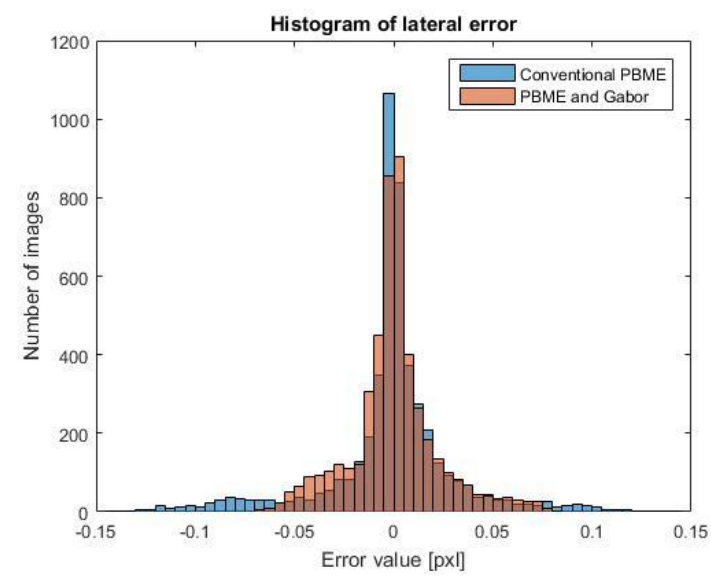

Fig. 4. Histogram of error [Lateral]. The highest errors corresponding with the edges of the histogram obtained with PBME method (blue) are eliminated by introducing our measure of confidence (orange).

The overall improvement in terms of Mean Square Error (MSE) was of $53.8 \%$ for the lateral displacement estimation and $73.6 \%$ for the axial displacement estimation. The comparison between the two methods (PBME with and without the measure of confidence) is presented in Table 2:

TABLE II. COMPARISON MSE FOR CONVENTIONAL PBME, PBME WITH MEASURE OF CONFIDENCE AND THE ACHIEVED IMPROVEMENT

\begin{tabular}{|l|l|l|l|}
\hline $\begin{array}{l}\text { MSE } \\
\text { Error/ } \\
\text { Direction }\end{array}$ & $\begin{array}{l}\text { Conventional } \\
\text { PBME }\end{array}$ & $\begin{array}{l}\text { PBME } \\
\text { With measure of } \\
\text { confidence }\end{array}$ & $\begin{array}{l}\text { Improvement after } \\
\text { using the measure }\end{array}$ \\
\hline Axial & $\begin{array}{l}0.0808 \mathrm{pxl} \\
(0.62 \mathrm{um})\end{array}$ & $\begin{array}{l}0.0213 \mathrm{pxl} \\
(0.16 \mathrm{um})\end{array}$ & $73.62 \%$ \\
\hline Lateral & $\begin{array}{l}0.0011 \mathrm{pxl} \\
(0.27 \mathrm{um})\end{array}$ & $\begin{array}{l}0.0004 \mathrm{pxl} \\
(0.12 \mathrm{um})\end{array}$ & $53.85 \%$ \\
& & & \\
\hline
\end{tabular}

\section{CONCLUSION}

Pattern orientation has been shown to be a reliable feature for the improvement of the PBME method applied to ultrafast US-TO images. The orthogonal pattern orientation present in images containing oscillations in both axial and lateral direction was detected using Gabor filters. A measure of confidence was introduced for PBME algorithm, by discarding the pixels classified by k-Means method as having orientations that do not follow the orthogonal directions of the beams. The approach proposed in this study significantly improved the robustness of the conventional estimator.

\section{ACKNOWLEDGMENT}

This project has received funding from the European Union's Horizon 2020 research and innovation programme under the Marie Skłodowska-Curie grant agreement No 642612. This work was performed within the framework of the LABEX PRIMES (ANR-11-LABX-0063) of Université de Lyon, within the program "Investissements d'Avenir" (ANR11-IDEX-0007) operated by the French National Research Agency (ANR).

\section{REFERENCES}

[1] M. Alessandrini, A. Basarab, H. Liebgott, and O. Bernard, "Cardiac motion assessment from echocardiographic image sequences by means of the structure multivector," in 2013 IEEE International Ultrasonics Symposium (IUS), 2013, pp. 1541-1544.

[2] J. A. Jensen and P. Munk, "A new method for estimation of velocity vectors.," IEEE Trans. Ultrason. Ferroelectr. Freq. Control, vol. 45, no. 3, pp. 837-51, 1998.

[3] M. E. Aderson, "Multi-dimensional velocity estimation with ultrasound using spatial quadrature," IEEE Trans. Ultrason. Ferroelectr. Freq. Control, vol. 45, no. 3, pp. 852-861, May 1998.

[4] H. Liebgott, J. Fromageau, J. E. Wilhjelm, D. Vray, and P Delachartre, "Beamforming Scheme for 2D Displacement Estimation in Ultrasound Imaging," EURASIP J. Adv. Signal Process., vol. 2005, no. 8, pp. 1212-1220, 2005.

[5] C. Sumi, "Multidimensional displacement vector measurement methods utilizing instantaneous phase.," Conf. Proc. ... Annu. Int Conf. IEEE Eng. Med. Biol. Soc. IEEE Eng. Med. Biol. Soc. Annu. Conf., vol. 2, pp. 1704-7, 2005.

[6] X. Guo, H. Liebgott, and D. Friboulet, "Back-propagation beamformer design for motion estimation in echocardiography.," Ultrason. Imaging, vol. 37, no. 3, pp. 179-204, Jul. 2015.

[7] M. Alessandrini, A. Basarab, L. Boussel, X. Guo, A. Serusclat, D. Friboulet, D. Kouamé, O. Bernard, and H. Liebgott, "A new technique for the estimation of cardiac motion in echocardiography based on transverse oscillations: a preliminary evaluation in silico and a feasibility demonstration in vivo.," IEEE Trans. Med. Imaging, vol. 33, no. 5, pp. 1148-62, May 2014.

[8] S. Salles, A. J. Y. Chee, D. Garcia, A. C. H. Yu, D. Vray, and H. Liebgott, "2-D arterial wall motion imaging using ultrafast ultrasound and transverse oscillations.," IEEE Trans. Ultrason. Ferroelectr. Freq. Control, vol. 62, no. 6, pp. 1047-58, Jun. 2015.

[9] J. A. Jensen, "Optimization of transverse oscillating fields for vector velocity estimation with convex arrays," in 2013 IEEE International Ultrasonics Symposium (IUS), 2013, pp. 1753-1756.

[10] A. Basarab, P. Gueth, H. Liebgott, and P. Delachartre, "Phase-based block matching applied to motion estimation with unconventional beamforming strategies.," IEEE Trans. Ultrason. Ferroelectr. Freq. Control, vol. 56, no. 5, pp. 945-57, May 2009.

[11] J. A. Jensen, "Field: A Program for Simulating Ultrasound Systems," Pap. Present. 10th Nord. Conf. Biomed. Imaging Publ. Med. Biol. Eng. Comput., vol. 34, no. sup. 1, pp. 351-353, 1996.

[12] J. A. Jensen and N. B. Svendsen, "Calculation of pressure fields from arbitrarily shaped, apodized, and excited ultrasound transducers," IEEE Trans. Ultrason. Ferroelectr. Freq. Control, vol. 39, no. 2, pp. 262-267, Mar. 1992.

[13] S. Salles, H. Liebgott, D. Garcia, and D. Vray, "Full 3-D transverse oscillations: a method for tissue motion estimation.," IEEE Trans. Ultrason. Ferroelectr. Freq. Control, vol. 62, no. 8, pp. 1473-85, Aug. 2015.

[14] G. Montaldo, M. Tanter, J. Bercoff, N. Benech, and M. Fink, "Coherent plane-wave compounding for very high frame rate ultrasonography and transient elastography.," IEEE Trans. Ultrason. Ferroelectr. Freq. Control, vol. 56, no. 3, pp. 489-506, Mar. 2009. 